\title{
NANOEMULSÕES ÓLEO DE LARANJA/ÁGUA PREPARADAS EM HOMOGENEIZADOR DE ALTA PRESSÃO
}

\section{Loretta R. Kourniatis, Luciana S. Spinelli e Claudia R. E. Mansur*}

Instituto de Macromoléculas Professora Eloisa Mano, Universidade Federal do Rio de Janeiro, Cidade Universitária, CT, Bl. J, Ilha do Fundão, 21945-970 Rio de Janeiro - RJ, Brasil

\section{Gaspar González}

Centro de Pesquisas e Desenvolvimento Leopoldo Américo Miguez de Mello, Cidade Universitária, Q. 7, Ilha do Fundão, 21949-900 Rio de Janeiro - RJ, Brasil

Recebido em 13/2/09; aceito em 22/7/09; publicado na web em 11/1/10

\begin{abstract}
ORANGE OIL/WATER NANOEMULSIONS PREPARED BY HIGH PRESSURE HOMOGENIZER. The objective of this work was to use the high-pressure homogenizer (HPH) to prepare stable oil/water nanoemulsions presenting narrow particle size distribution. The dispersions were prepared using nonionic surfactants based on ethoxylated ether. The size and distribution of the droplets formed, along with their stability, were determined in a Zetasizer Nano ZS particle size analyzer. The stability and the droplet size distribution in these systems do not present the significant differences with the increase of the processing pressure in the HPH). The processing time can promote the biggest dispersion in the size of particles, thus reducing its stability.
\end{abstract}

Keywords: nanoemulsions; high-pressure homogenizer; orange oil.

\section{INTRODUÇÃO}

Nanoemulsões são dispersões onde o tamanho das gotas dispersas estão em escala nanométrica, na qual a faixa de tamanho está compreendida entre 10 e 100 nanômetros. ${ }^{1}$ Este pequeno tamanho das gotas confere sua estabilidade, evitando a sedimentação (cremeação). A maturação de Ostwald, também conhecida como envelhecimento de Ostwald, é o mecanismo principal para a desestabilização de nanoemulsões, o qual surge da polidispersão de uma emulsão e da diferença de solubilidade e/ou dos potenciais químicos entre as partículas pequenas e grandes.,3

Para formar nanoemulsões é necessária uma contribuição de energia, geralmente, de dispositivos mecânicos ou do potencial químico dos componentes. ${ }^{4}$ Mecanicamente, uma alta energia necessária para promover uma taxa de cisalhamento capaz de deformar a partícula, geralmente, é alcançada através de homogeneizadores de alta pressão ou geradores de ultrassom. A aplicação de alta energia gera forças que podem romper as gotas da fase dispersa, de forma que a diferença entre as pressões interna e externa da gota seja superada (Lei de Young-Laplace). ${ }^{5}$

Basicamente, um homogeneizador de alta pressão consiste de um gerador de alta pressão e um dispositivo de interação. O líquido processado passa sob alta pressão por uma seção convergente e então se expande. ${ }^{6,7}$ De acordo com a geometria deste dispositivo de interação, os homogeneizadores de alta pressão podem ser divididos, principalmente, em duas categorias: equipamentos com uma válvula em forma de anel e equipamentos baseados em uma câmara de interação entre dois líquidos. ${ }^{8}$

Os homogeneizadores com válvula em forma de anel incluem os homogeneizadores de AVP-Gaulin e o Stansted, onde uma válvula hidráulica ou eletrônica assegura uma constante e reprodutível pressão durante a homogeneização. A diferença entre estes dois equipamentos está relacionada à geometria das válvulas de entrada e saída da amostra. Devido a essas diferenças de geometria, o homogeneizador Stansted permite que se alcance uma homogeneização com uma maior pressão (até $50000 \mathrm{psi}$ ). ${ }^{9}$

*e-mail: celias@ima.ufrj.br
A operação com este tipo de homogeneizador ocorre em duas fases, envolvendo dois mecanismos principais: cavitação e cisalhamento durante o fluxo laminar e turbulento. Na primeira fase, a dispersão é forçada a passar através de alta pressão com alta velocidade por um orifício anular estreito. Devido à pressão, a válvula se abre contra uma mola. A segunda fase ocorre paralelamente com a primeira: a uma alta velocidade de fluxo, a pressão estática no fluido é alterada. Se esta atingir valores próximos da pressão de vapor, surgirão bolhas cheias de vapor ou gás (cavitação) que aumentarão até que haja a implosão das mesmas. Dessas implosões, resulta uma alta tensão local que provoca a quebra e o rompimento das gotas, gerando a formação de novas gotas com tamanhos menores. ${ }^{8}$

Para os homogeneizadores com câmaras de interação, a turbulência ocorrida durante o fluxo, e não a cavitação, é responsável pelo rompimento das gotas. Um exemplo desses equipamentos é o Microfluidizer, no qual a homogeneização ocorre dentro de uma câmara de interação onde o fluxo líquido é guiado através de microcanais para uma área de convergência. O fluxo processado é liberado por uma bomba pneumática que é capaz de pressurizar o ar comprimido (80-100 psi) para uma alta velocidade e pressão (10000 psi). Quando o fluxo em alta pressão entra na câmara de interação, ocorre uma colisão e um alto cisalhamento, resultando em partículas em escala nanométrica, dispersões estáveis ou emulsões. ${ }^{8,10}$

Neste trabalho, nanoemulsões de óleo em água foram preparadas, em presença de tensoativo não-iônicos, em um homogeneizador de alta pressão (HPH). Os seguintes parâmetros foram avaliados: pressão de processamento, tempo de processamento, tipo de óleo e hidrofilicidade do tensoativo. Para tanto, foram selecionados como fase oleosa o óleo essencial de laranja e o terpeno de laranja, o qual, segundo a literatura, ${ }^{11}$ constitui cerca de $96 \%$ a composição química deste óleo essencial.

\section{PARTE EXPERIMENTAL}

\section{Materiais}

Os tensoativos não-iônicos usados, doados pela Empresa Oxiteno - Brasil, são do tipo éter laurílico etoxilado (linha Unitol L) e éter 
oléico etoxilado (linha Unitol O), os quais apresentam quantidades diferentes de unidades de óxido de etileno (EO) em suas cadeias.

As fases oleosas das emulsões o/a foram compostas por óleo essencial de laranja e terpeno de laranja, ambos de procedência All Flavors - Brasil. Água destilada e deionizada foi usada como fase aquosa.

\section{Métodos}

Análise de ressonância magnética nuclear de hidrogênio $\left(R M N-{ }^{1} H\right)$ dos tensoativos

As análises de $\mathrm{RMN}-{ }^{1} \mathrm{H}$ foram realizadas no espectrômetro Varian 300 , operando a $300 \mathrm{MHz}$ e $30 \pm 0,1^{\circ} \mathrm{C}$. Clorofórmio deuterado foi usado como solvente. $\mathrm{O}$ número de unidades de EO presente nas cadeias dos tensoativos foi calculado com base nas áreas dos deslocamentos químicos localizados em $0,83 \mathrm{ppm}$ (relativo aos hidrogênios do $\mathrm{CH}_{3}$ terminal localizado na parte hidrocarbônica da estrutura do tensoativo) e 3,5 ppm (relativo aos hidrogênios dos grupos $\mathrm{CH}_{2}$ do EO e do grupo $\mathrm{CH}_{2}$ da parte hidrocarbônica, próximo ao grupo EO).

\section{Determinação do valor de HLB das fases oleosas}

A determinação do valor do balanço hidrófilo-lipófilo (HLB) das fases oleosas foi realizada por meio do método direto, o qual compara visualmente o comportamento de tensoativos com padrões de HLB conhecidos. ${ }^{12}$

Foram preparadas $50 \mathrm{~g}$ de dispersões, formadas pelas soluções aquosas das misturas de tensoativos Unitol L100/Unitol L20 e o óleo. As concentrações em massa foram fixas: $5 \%$ da mistura de tensoativos, $10 \%$ de óleo e $85 \%$ de água. As soluções aquosas foram preparadas utilizando-se quantidades de tensoativos apropriadas para a obtenção de soluções com valores de HLB conhecidos e compreendidos na faixa de 6,4 a 11,0, calculadas com auxílio da Equação 1.

$H L B_{d}=\left(M_{a} H L B_{a}+M_{b} H L B_{b}\right) / M_{T}$

$M_{a}$ - massa de tensoativo a $(\mathrm{g}) ; M_{b}-$ massa de tensoativo b $(\mathrm{g}) ; M_{T}-$ massa total de tensoativo (g); $H L B_{d}$ - HLB desejado; $H L B_{a}$ - valor de HLB do tensoativo a; $H L B_{b}$ - valor de HLB do tensoativo b.

As dispersões foram obtidas adicionando-se primeiramente os tensoativos na fase aquosa, sendo solubilizados por um período de aproximadamente $24 \mathrm{~h}$. Posteriormente, estas soluções foram adicionadas na fase oleosa, sob agitação magnética constante por cerca de 20 min e estes sistemas foram deixados em repouso por cerca de $24 \mathrm{~h}$.

Após o repouso, as dispersões foram avaliadas visualmente, sendo o valor de HLB do óleo correspondente ao valor de HLB da dispersão que apresentou a maior estabilidade, ou seja, que apresentou a menor separação de fases.

\section{Análise de tensão interfacial óleo/solução aquosa de tensoativo}

Os valores de tensão interfacial óleo/solução aquosa de tensoativo (em $\mathrm{mN} / \mathrm{m}$ ) foram medidos usando o tensiômetro digital Krüss, modelo K10, o qual é baseado no método do anel de Du Noüy, a temperatura de $25^{\circ} \mathrm{C}$.

Soluções aquosas dos tensoativos, nas concentrações de 8,10 e $12 \% \mathrm{~m} / \mathrm{m}$, foram preparadas sem qualquer diluição posterior. Todas as medidas foram feitas, pelo menos, em triplicata.

\section{Preparação das emulsões óleo/água no equipamento $\mathrm{HPH}$}

$\mathrm{O}$ equipamento utilizado para o preparo das emulsões o/a foi o homogeneizador de alta pressão Emulsiflex C5, no qual a homogeneização ocorre dentro de uma câmara de interação. Devido à falta de homogeneidade do fluxo, é geralmente necessário passar este fluido diversas vezes através do dispositivo até se obter uma faixa de tamanho de partículas adequada. Este procedimento é denominado de ciclos de processamento.

Antes da preparação de emulsões o/a foram determinados os tempos de cada ciclo de processamento, em segundos, da passagem de um volume definido de amostra, a uma determinada pressão.

Inicialmente foram produzidas emulsões o/a na ausência do tensoativo. Para tanto, foram preparadas $30 \mathrm{~g}$ de dispersões contendo fase oleosa nas concentrações de 5, 10 e $15 \% \mathrm{~m} / \mathrm{m}$. Em presença de tensoativo, as emulsões o/a foram preparadas contendo concentração de fase oleosa fixa de 15 e $10 \%$ em massa dos tensoativos não-iônicos.

\section{Formação e estabilidade das nanoemulsões}

As emulsões de o/a produzidas foram caracterizadas utilizando o analisador de tamanho de partículas Zetasizer Nano ZS, Malvern, a fim de se obter a faixa de tamanho das gotas dispersas na emulsão, bem como a sua distribuição de tamanho. Este equipamento apresenta o detector em uma posição a $173^{\circ}$ do feixe de luz incidente, sendo conhecido como detecção por retroespalhamento, tecnologia patenteada conhecida como NIBS (retroespalhamento não invasivo).

A estabilidade destas emulsões o/a foi também avaliada por meio do monitoramento do tamanho e da distribuição de tamanho das partículas em função do tempo. Os tempos usados neste estudo foram: ao término do preparo da emulsão (tempo 0), e após 1, 2, 24, 48,72 h e, assim sucessivamente, até a observação visual da completa separação de fases do sistema.

Os resultados obtidos no analisador de tamanho de partícula são mostrados em forma de gráficos de volume como função do tamanho das partículas produzidas em cada emulsão. Os valores de índice de refração foram medidos em refratômetro ABBE, Bausch \& Lomb e a viscosidade utilizada nas medidas foi do solvente água.

\section{RESULTADOS E DISCUSSÃO}

A caracterização dos tensoativos quanto ao número de unidades de $\mathrm{EO}$ em suas cadeias, determinados por $\mathrm{RMN}-{ }^{1} \mathrm{H}$, e os valores de HLB destes tensoativos, informados pela empresa doadora, são mostrados na Tabela 1.

Tabela 1. Tensoativos da linha Unitol ${ }^{\circledR} \mathrm{L}$ e Unitol ${ }^{\circledR} \mathrm{O}$

\begin{tabular}{|c|c|c|}
\hline Nome & Estruturas ${ }^{(a)}$ & Valores de HLB $^{\text {(b) }}$ \\
\hline Unitol L ${ }^{\circledR} 20$ & $\begin{array}{c}\text { Éter laurílico, com } 2 \\
\text { unidades de EO }\end{array}$ & 6,4 \\
\hline Unitol L ${ }^{\circledR} 60$ & $\begin{array}{c}\text { Éter laurílico com } 6 \\
\text { unidades de EO }\end{array}$ & 11,5 \\
\hline Unitol L ${ }^{\circledR} 100$ & $\begin{array}{c}\text { Éter laurílico com } 10 \\
\text { unidades de EO }\end{array}$ & 13,9 \\
\hline Unitol $\mathrm{L}^{\circledR} 230$ & $\begin{array}{c}\text { Éter laurílico com } 23 \\
\text { unidades de EO }\end{array}$ & 16,9 \\
\hline Unitol $\mathrm{O}^{\circledR} 100$ & $\begin{array}{c}\text { Éter oleico com } 10 \\
\text { unidades de EO }\end{array}$ & 12,4 \\
\hline
\end{tabular}

(a) Determinado por ressonância magnética nuclear de hidrogênio (RMN$\left.{ }^{1} \mathrm{H}\right)$; (b) dados fornecidos pela Empresa Oxiteno

\section{Determinação dos valores de HLB das fases oleosas}

Os valores de HLB das fases oleosas utilizadas (óleo de laranja e terpeno de laranja) foram determinados pelo método visual a partir da formação e avaliação da estabilidade de emulsões o/a obtidas em presença das misturas de tensoativos Unitol L100/Unitol L20.

As dispersões terpeno de laranja/água apresentaram separação de fases em toda a faixa de HLB analisada $(6,4$ a 11,0). Porém, a 
emulsão preparada com o tensoativo Unitol L20 puro apresentou uma relativa homogeneidade, indicando que o valor do HLB do terpeno de laranja é próximo a 6,4.

As emulsões óleo de laranja/água apresentaram relativa homogeneidade no intervalo de HLB compreendido entre 8,5 e 9,0. Avaliando-se o comportamento das emulsões dentro deste intervalo de HLB, a emulsão que apresentou a maior homogeneidade foi aquela preparada com a mistura de tensoativos de HLB 8,7. Portanto, pode ser considerado que o valor de HLB do óleo de laranja utilizado neste trabalho é igual a 8,7 .

O valor de HLB mais alto obtido para o óleo de laranja, quando comparado ao valor de HLB do terpeno de laranja determinado ( $\approx 6,4)$, era esperado tendo em vista que, segundo a literatura, ${ }^{11}$ na composição deste óleo, além do d-limoneno, estão presentes outros compostos polares, tais como os derivados oxigenados do terpeno, mirceno, octanal, linalol, entre outros, os quais estariam elevando seu valor de HLB.

\section{Análise de tensão interfacial óleo/água}

As interfaces óleo de laranja e terpeno de laranja com as soluções aquosas dos tensoativos Unitol L20, Unitol L60, Unitol L100, Unitol L230 e Unitol O100 foram analisadas em concentrações de: 8, 10 e 12 $\% \mathrm{~m} / \mathrm{m}$, com a finalidade de avaliar o comportamento dos tensoativos nas interfaces formadas entre a água com os diferentes óleos e nas concentrações usadas para preparar as emulsões, de acordo com o que está apresentado na Tabela 2.

Analisando-se os valores de tensão interfacial o/a na ausência de tensoativos, pode ser observado que, como esperado, o maior valor foi obtido na interface formada pela água com o terpeno de laranja seguido pelo do óleo de laranja, o qual é o óleo de caráter menos hidrófobo (HLB=8,7).

Todos os tensoativos utilizados reduziram as tensões interfaciais o/a para valores muito baixos. Os tensoativos que promoveram a maior redução nestes valores foram aqueles que apresentaram os menores teores de unidades de EO em suas cadeias (Unitol L20 e Unitol L60). Por outro lado, o tensoativo Unitol L230, mais hidrófilo, foi o que apresentou a menor atividade nas interfaces o/a formadas. Comparando-se os resultados obtidos com os tensoativos Unitol L100 e Unitol O100 observa-se que a diferença nos valores é atribuída ao maior tamanho da cadeia hidrófoba no tensoativo Unitol O100, já que ambos contêm teores de unidades de EO semelhantes em suas cadeias.

Este comportamento está de acordo com a literatura, ${ }^{13}$ onde os tensoativos não-iônicos derivados de EO (óxido de etileno condensado de álcoois hexadecanol e dodecanol) apresentam menores áreas na interface água/ar quando se analisam os derivados de hexadecanol, apesar da diferença ser pequena, ou seja, a área ocupada por uma molécula de tensoativo diminui com a redução do tamanho da cadeia de EO, o que acarreta em um maior empacotamento na interface. Segundo a isoterma de adsorção de Gibbs, ${ }^{14}$ quanto menor esta área, maior será a redução da tensão interfacial.

\section{Formação de emulsões no equipamento HPH}

As condições de processamento do homogeneizador de alta pressão (HPH), tais como pressão, tempo e número de ciclos, foram primeiramente avaliadas para posterior utilização destas condições na produção das nanoemulsões, em presença dos tensoativos.

A pressão de processamento máxima no equipamento utilizado neste trabalho foi de 25000 psi. Porém, em pressões muito elevadas, a vazão de saída da amostra homogeneizada foi muito baixa, o que tornava seu uso inviável. Sendo assim, a pressão mais alta em que foi possível gerar uma maior vazão dos fluidos foi de 17500 psi. Neste estudo, então, foram selecionadas para os experimentos as pressões de 5000, 10000 e 17500 psi, as quais equivalem à pressão baixa, média e alta neste equipamento.

Para controlar o número de ciclos de processamento se fez necessário realizar as medidas dos tempos de um ciclo, ou seja, o tempo que uma alíquota de amostra leva para percorrer todo o sistema do homogeneizador em uma dada pressão na bomba. Esses tempos foram determinados com a passagem de $30 \mathrm{~mL}$ de água destilada e deionizada, nas pressões de 5000, 10000 e 17500 psi, sendo de respectivamente, 24,31 e $52 \mathrm{~s}$. Ou seja, para se obter quatro ciclos de processamento, a dispersão é forçada a passar por todo equipamento no tempo 4 vezes maior do que o tempo de um ciclo, medido naquela determinada pressão e volume de dispersão.

\section{Preparação das emulsões o/a na ausência de tensoativo}

As condições de processamento utilizadas no preparo das dispersões, contendo somente fase oleosa e água, foram: pressões de 5000, 10000 e 17500 psi; número de ciclos de 1 a 4; e concentrações de fase oleosa de 5, 10 e $15 \%$ em uma massa total de $30 \mathrm{~g}$ de emulsão.

Todas as emulsões o/a preparadas foram caracterizadas quanto ao tamanho de partículas da fase dispersa. As Figuras 1a e 1b mostram as curvas de distribuição de tamanho das fases oleosas óleo de laranja e terpeno de laranja, respectivamente, na concentração de fase oleosa de $10 \% \mathrm{~m}$. Estes gráficos foram construídos de modo que as curvas

Tabela 2. Valores das tensões interfaciais óleo/solução aquosa dos tensoativos

\begin{tabular}{|c|c|c|c|c|c|}
\hline \multirow{2}{*}{ Tensoativo } & \multirow{2}{*}{ Fase oleosa (a) } & \multicolumn{4}{|c|}{ Tensão interfacial (mN/m) } \\
\hline & & $0 \% \mathrm{~m} / \mathrm{m}(\mathrm{b})$ & $8 \% \mathrm{~m} / \mathrm{m}(\mathrm{b})$ & $10 \% \mathrm{~m} / \mathrm{m}(\mathrm{b})$ & $12 \% \mathrm{~m} / \mathrm{m}(\mathrm{b})$ \\
\hline \multirow[t]{2}{*}{ Unitol L20 } & Óleo de laranja & 5,4 & 1,0 & (c) & (c) \\
\hline & Terpeno de laranja & 15,5 & (c) & (c) & (c) \\
\hline \multirow[t]{2}{*}{ Unitol L60 } & Óleo de laranja & 5,4 & (c) & (c) & (c) \\
\hline & Terpeno de laranja & 15,5 & 1,1 & 1,1 & 1,3 \\
\hline \multirow[t]{2}{*}{ Unitol L100 } & Óleo de laranja & 5,4 & (c) & (c) & (c) \\
\hline & Terpeno de laranja & 15,5 & 1,9 & 1,8 & 1,7 \\
\hline \multirow[t]{2}{*}{ Unitol L230 } & Óleo de laranja & 5,4 & 1,8 & 1,8 & 1,8 \\
\hline & Terpeno de laranja & 15,5 & 4,3 & 4,1 & 4,0 \\
\hline \multirow[t]{2}{*}{ Unitol O100 } & Óleo de laranja & 5,4 & 1,6 & 1,2 & 1,3 \\
\hline & Terpeno de laranja & 15,5 & 2,1 & 2,2 & 2,2 \\
\hline
\end{tabular}

(a) Relação de fase aquosa/fase oleosa constante de 1:1; (b) concentração de tensoativo na dispersão; (c) tensões muito baixas (< 1,0 mN/m) 
obtidas na pressão mais baixa (5000 psi) estejam localizadas na parte inferior do gráfico, seguidos dos resultados obtidos nas pressões de 10000 e de 17500 psi. Além disso, o número de ciclos utilizado no processamento das dispersões está indicado ao lado de cada valor de pressão mostrado na legenda dos gráficos. Por exemplo, na pressão de 5000 psi, com um (1) ciclo de processamento, a curva foi denominada de $5000 \mathrm{psi} / 1 \mathrm{c}$.
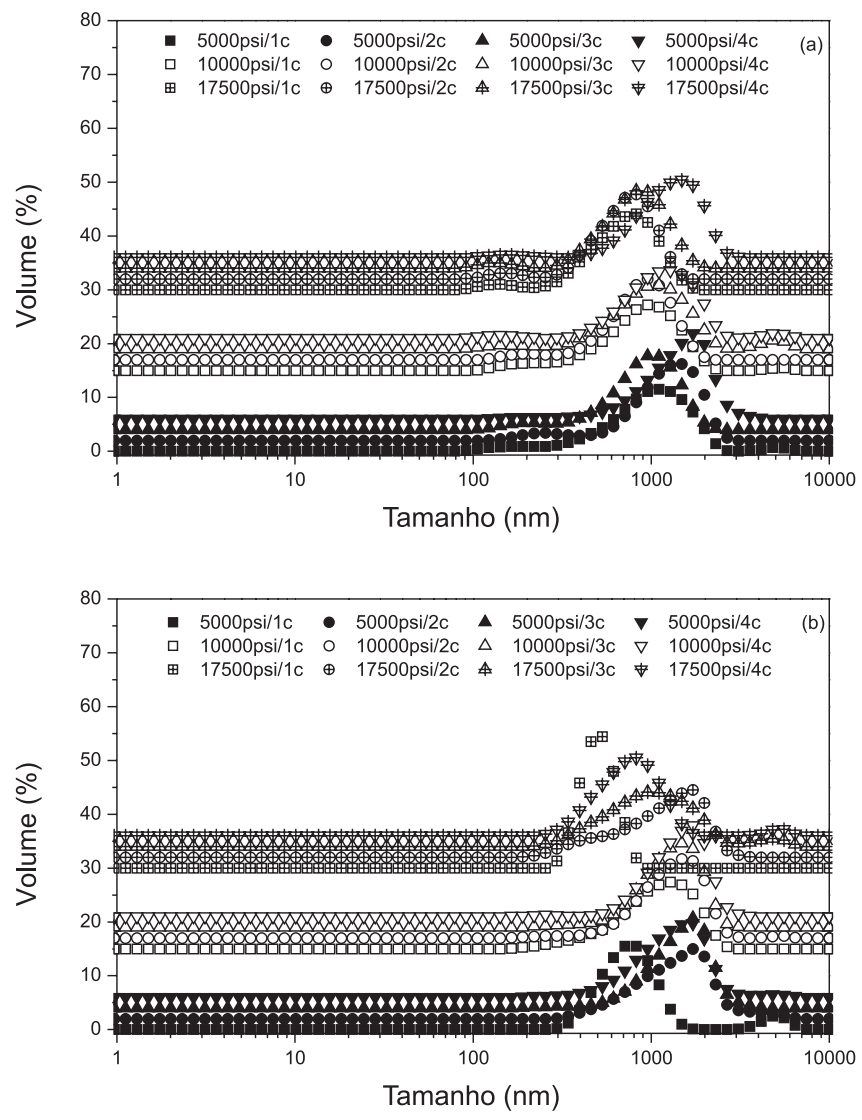

Figura 1. Distribuição do tamanho das partículas das emulsões óleo de laranjalágua(a) e terpeno de laranja/água(b) preparadas em diferentes pressões de processamento no $\mathrm{HPH}(5000,10000$ e 17500 psi). Concentração de fase oleosa: $10 \% \mathrm{~m} / \mathrm{m}$

Ao término do processamento de todas as dispersões no HPH foram observadas pequenas concentrações de óleo residual no fundo do cilindro de amostra, mostrando que, devido à baixa estabilidade das emulsões formadas, estas apresentavam uma concentração de óleo ligeiramente inferior à concentração inicial de entrada no equipamento. Este comportamento foi principalmente observado para as dispersões preparadas com os óleos essenciais na concentração de fase oleosa de $15 \% \mathrm{~m} / \mathrm{m}$ e, por este motivo, não foram realizadas as análises de distribuição de tamanho de partículas destas dispersões.

No processamento das dispersões à base de óleo de laranja, nas concentrações de 5 e $10 \% \mathrm{~m} / \mathrm{m}$ de fase oleosa, foi observado o mesmo comportamento: em nenhuma pressão empregada houve a formação de nanoemulsões na ausência de tensoativo. As emulsões obtidas apresentaram tamanhos médios de partículas em torno de $1000 \mathrm{~nm}$ e distribuição destes tamanhos entre 300 e $3000 \mathrm{~nm}$. Estes tamanhos médios foram ligeiramente reduzidos quando foram utilizadas mais altas pressões (17500 psi), o tamanho médio das partículas passou a ser de $800 \mathrm{~nm}$, ao invés do valor de $1000 \mathrm{~nm}$, obtido em 5000 psi, como pode ser observado nas curvas mostradas na Figura 1a.

As dispersões à base de terpeno de laranja apresentaram com- portamento semelhante ao observado na Figura 1a: as emulsões obtidas nas pressões de 5000 e 10000 psi apresentaram uma larga distribuição de tamanhos (entre 300 e 3000 nm). No entanto, com o aumento da pressão de processamento para 17500 psi, a emulsão formada apresentava-se com uma faixa de tamanho de partículas menor (compreendida entre 300 e $1000 \mathrm{~nm}$ ) e tamanho médio de 500 $\mathrm{nm}$, nos menores números de ciclos. As emulsões obtidas em maiores tempos de processamento apresentaram uma maior quantidade de óleo residual, ou seja, uma maior separação de fases (Figura 1b).

De forma geral, todas as emulsões formadas apresentaram separação de fases em tempos muito pequenos (menores que $60 \mathrm{~min}$ ) ao final do processamento no HPH.

\section{Preparação das emulsões o/a em presença do tensoativo}

Na formação de nanoemulsões empregando a emulsificação de alta energia, o tensoativo é utilizado para que ocorra uma melhor homogeneização entre o óleo e água e a emulsão permaneça estável por um longo período de tempo. Para isso, eles promovem a redução nas tensões interfaciais o/a e também promovem a estabilização estérica da fase dispersa. Assim, os processos de desestabilização naturais que ocorrem em emulsões (maturação de Ostwald e coalescência) são retardados., ${ }^{2,3,15}$

A caracterização das emulsões o/a obtidas foi realizada em analisador de tamanho de partículas, sendo todas as análises realizadas em triplicata. Portanto, nos gráficos obtidos, são apresentadas as curvas médias de distribuição de tamanho de partículas das emulsões, com as respectivas barras de erro.

As emulsões o/a foram preparadas em presença dos tensoativos comerciais puros (Unitol L60, Unitol L100, Unitol L230 e Unitol O100) utilizando para seu processamento as pressões baixa, média e alta $(5000,10000$ e 17500 psi) e o número de ciclos igual a 3. Este número de ciclos foi selecionado tendo em vista os resultados obtidos anteriormente (Figura 1). A concentração de fase oleosa foi constante em todos os sistemas e igual a $15 \% \mathrm{~m} / \mathrm{m}$, visando a produção de emulsões em concentrações mais altas de fase dispersa. A concentração de tensoativo foi de $10 \% \mathrm{~m} / \mathrm{m}$, na qual todos os sistemas apresentam baixos valores de tensão interfacial, como mostrado na Tabela 2 .

Não foi possível preparar emulsões utilizando o tensoativo Unitol L20 puro devido a sua difícil solubilização em água, por conter a menor cadeia polar (composta por duas unidades de EO) em sua estrutura.

As Figuras 2 a 4 mostram os gráficos obtidos de distribuição de tamanho de partículas das emulsões óleo de laranja/água contendo os tensoativos Unitol L60, Unitol L100 e Unitol L230, respectivamente. A partir destes resultados pode ser observado que a formação de nanoemulsões com distribuição de tamanho monomodal (com uma única faixa de tamanho de partículas, compreendida entre 10 e $60 \mathrm{~nm}$ ) foi obtida apenas ao ser utilizado o tensoativo Unitol L60 (Figura 2), a qual foi independente da pressão utilizada no processamento. As emulsões óleo de laranja/água formadas com os tensoativos Unitol L100 e Unitol L230 (Figuras 3 e 4, respectivamente) apresentaram distribuições de tamanho de partícula heterogêneas. Além disso, os maiores tamanhos de partículas de fase dispersa foram obtidos para as emulsões preparadas com o tensoativo Unitol L230 (Figura 4).

O menor valor de tensão interfacial deste sistema obtido em presença do tensoativo Unitol L60 deve ter sido a causa da formação de uma nanoemulsão. Por outro lado, o tensoativo Unitol L230, por conter em sua estrutura uma cadeia maior de EO, não causa uma redução no valor da tensão interfacial suficiente para a obtenção de sistemas contendo partículas dispersas de tamanhos reduzidos.

Emulsões utilizando-se o tensoativo Unitol O100 em sua formulação foram também preparadas (Figura 5). Este tensoativo apresenta como parte apolar uma cadeia hidrocarbônica contendo 18 átomos 


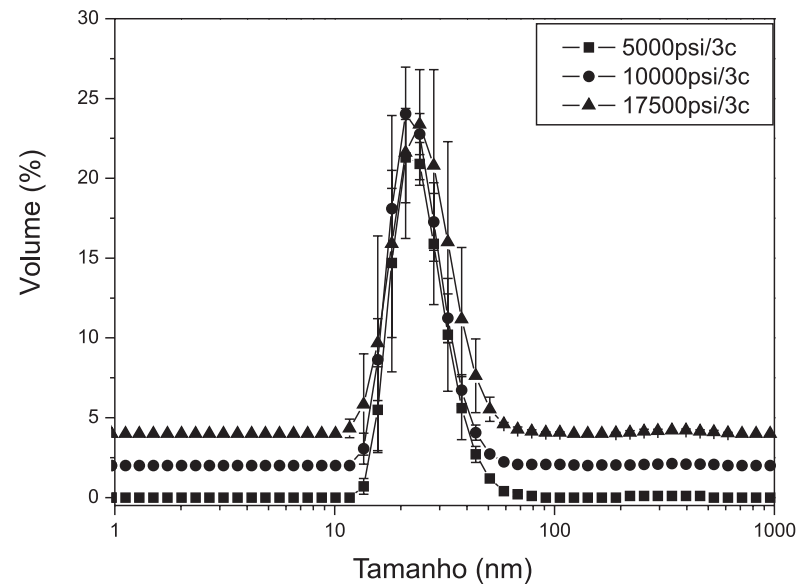

Figura 2. Distribuição do tamanho das partículas das emulsões óleo de laranja/água, preparadas em HPH, nas três diferentes pressões (5000, 10000, 17500 psi), utilizando-se o tensoativo Unitol L60, na concentração de 10 $\% \mathrm{~m} / \mathrm{m}$. Número de ciclos igual a 3

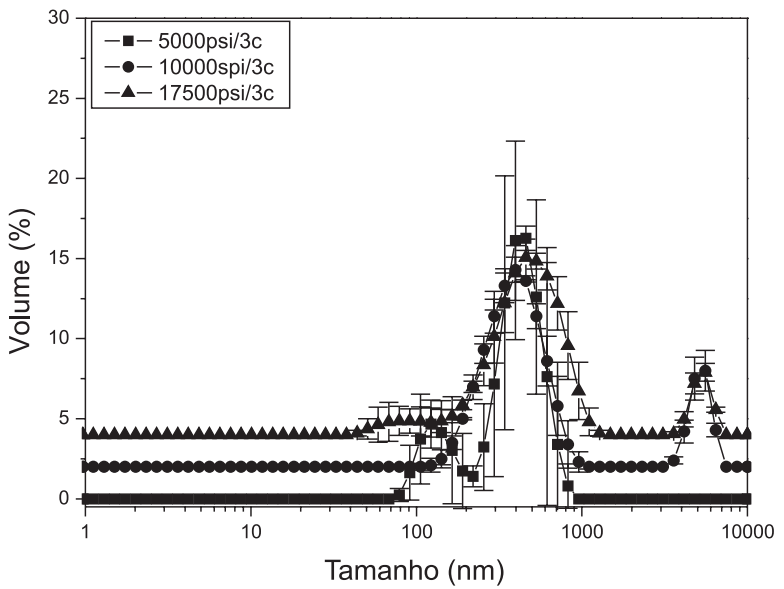

Figura 3. Distribuição do tamanho das partículas das nanoemulsões à base de óleo de laranja preparadas em HPH, nas três diferentes pressões $(5000$, 10000, 17500 psi), utilizando-se Unitol L100 na concentração de $10 \% \mathrm{~m} / \mathrm{m}$. Número de ciclos igual a 3

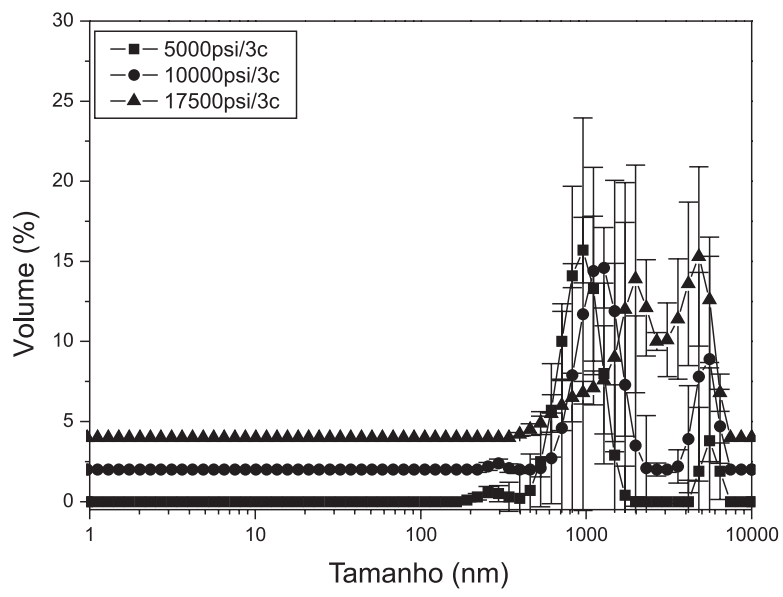

Figura 4. Distribuição do tamanho das partículas das nanoemulsões à base de óleo de laranja preparadas em HPH, nas três diferentes pressões $(5000$, 10000, 17500 psi), utilizando-se Unitol L230 na concentração de $10 \% \mathrm{~m} / \mathrm{m}$. Número de ciclos igual a 3 de carbono e como parte polar cadeias contendo 10 unidades de EO. Os gráficos de distribuição de tamanho de partículas obtidos para as emulsões à base de óleo de laranja, apresentaram distribuição estreita de tamanho (faixa compreendida entre 10 e $60 \mathrm{~nm}$ ), nas três concentrações utilizadas de tensoativo. Porém, em todos os casos, foram observados pequenos volumes de partículas maiores presentes nas emulsões.

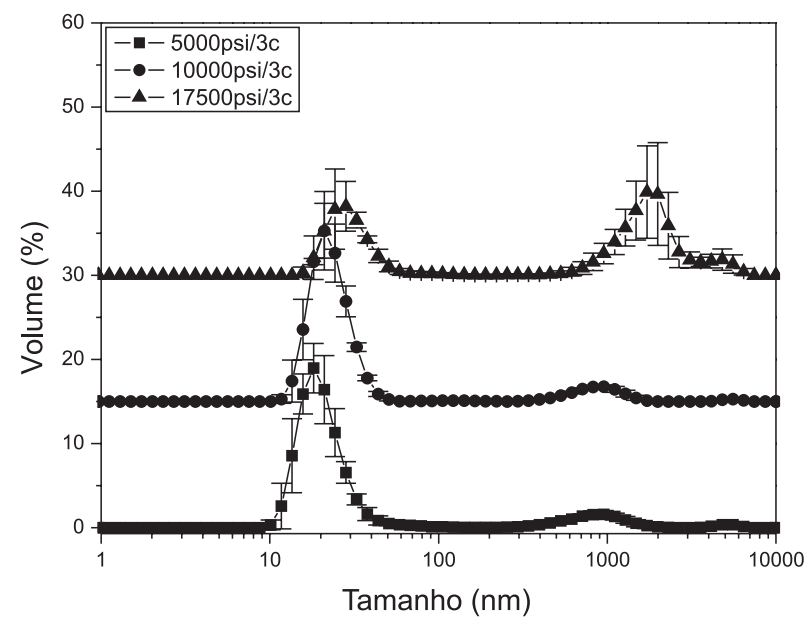

Figura 5. Distribuição do tamanho das partículas das nanoemulsões à base de óleo de laranja preparadas em HPH, nas três diferentes pressões (5000, 10000 e 17500 psi), utilizando-se Unitol O100 na concentração de $10 \% \mathrm{~m} / \mathrm{m}$. Número de ciclos igual a 3

A influência do tamanho da cadeia da parte apolar do tensoativo na obtenção de nanoemulsões pode ser avaliada por meio da comparação dos resultados mostrados nas Figuras 3 e 5 . Os tensoativos utilizados no preparo destas emulsões óleo de laranja/água (Unitol L100 e Unitol O100) reduziram a tensão interfacial destes sistemas de $5,4 \mathrm{mN} / \mathrm{m}$ para valores em torno de $1 \mathrm{mN} / \mathrm{m}$ (Tabela 2), porém esta redução foi mais pronunciada com o tensoativo Unitol O100. Por este motivo, os sistemas processados em presença do tensoativo de maior cadeia apolar (Unitol O100), apresentaram menores tamanhos de partículas (Figura 5).

Nanoemulsões também foram obtidas à base de terpeno de laranja, porém todos os sistemas se mostraram mais difíceis de serem processados, apresentando uma separação de fases muito rápida, devido ao caráter mais hidrófobo deste óleo.

\section{Avaliação da estabilidade das emulsões o/a}

A estabilidade de todas as emulsões o/a obtidas foi avaliada quanto à distribuição de tamanho das gotas formadas em função do tempo de preparo. Esta foi realizada analisando-se inicialmente a distribuição de tamanhos das partículas dispersas da emulsão tão logo ela tivesse sido processada no HPH (tempo zero). A seguir, a emulsão foi deixada em repouso e novas análises foram realizadas de tempos em tempos, até a observação da separação de fases destes sistemas.

Estas análises foram realizadas somente para as emulsões que apresentaram uma distribuição homogênea e estreita de tamanho das partículas formadas. As demais emulsões, por apresentarem mais larga distribuição nestes tamanhos, perderam sua estabilidade rapidamente.

Nestas emulsões, os tensoativos Unitol L100 (Figura 3), Unitol L230 (Figura 4) e Unitol O100 (Figura 5) não formaram emulsões estáveis; apesar do tensoativo Unitol O100 ter formado nanoemulsões com distribuições de tamanho estreita, esta não se apresentava totalmente homogênea, levando a uma rápida separação de fases. Como 
esperado, nanoemulsões óleo de laranja/água estáveis foram obtidas quando foi utilizado o tensoativo puro Unitol L60, na concentração de $10 \% \mathrm{~m} / \mathrm{m}$. A Figura 6 apresenta os resultados obtidos para as nanoemulsões óleo de laranja/água utilizando o tensoativo Unitol L60, na concentração de $10 \% \mathrm{~m} / \mathrm{m}$, preparadas sob a pressão de 5000 psi. Nesta e nas demais pressões utilizadas (10000 e 17500 psi) as nanoemulsões permaneceram estáveis por um período de 19 dias.

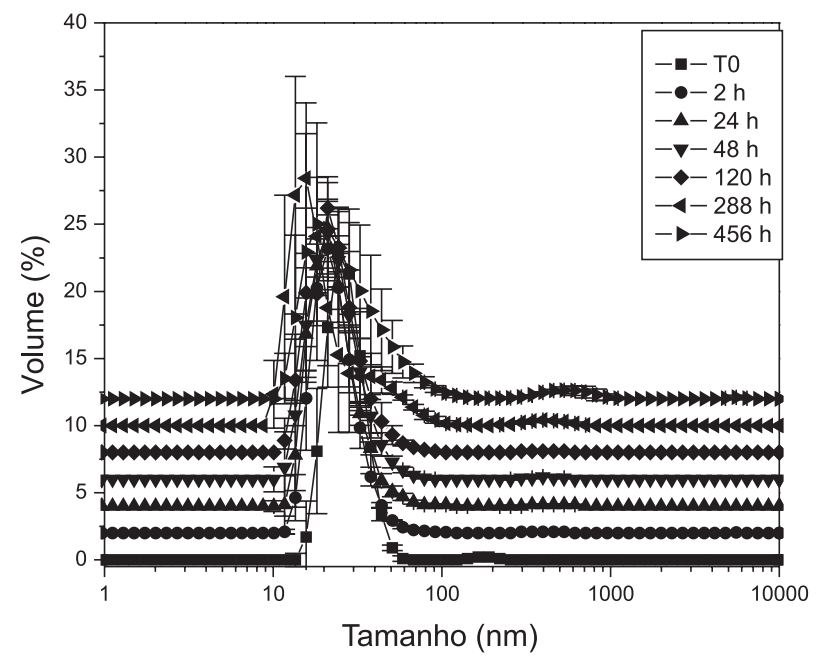

Figura 6. Distribuição do tamanho das partículas das emulsões óleo de laranjalágua, em função do tempo de preparo no $\mathrm{HPH}$, utilizando-se o tensoativo Unitol L60, na concentração de $10 \% \mathrm{~m} / \mathrm{m}$. Pressão de 5000 psi e número de ciclos igual a 3

Como já mencionado anteriormente, para os sistemas preparados à base de terpeno de laranja como fase oleosa, em presença dos tensoativos, foram produzidas nanoemulsões que não apresentaram nenhuma estabilidade. Estas emulsões apresentaram difícil interação água/óleo, havendo uma rápida separação de fases, muitas vezes, ainda dentro do cilindro de amostra do equipamento HPH.

\section{CONCLUSÕES}

O homogeneizador de alta pressão (HPH) utilizado neste trabalho, o qual possui a geometria do dispositivo de interação com uma válvula em forma de anel, mostrou ser um equipamento capaz de produzir nanoemulsões o/a estáveis, cuja fase oleosa é constituída por óleo de laranja, em presença do tensoativo não iônico à base de éter lauril etoxilado Unitol L60.
Foi observado que o aumento da pressão de processamento no HPH (de 5000 a 17500 psi) causa uma pequena variação no tamanho das partículas dispersas nos sistemas o/a. Por outro lado, foi observado nas emulsões preparadas na ausência do tensoativo que o tempo de processamento pode promover a maior dispersão no tamanho das partículas reduzindo, assim, sua estabilidade.

\section{AGRADECIMENTOS}

À Empresa Oxiteno do Brasil pela doação das amostras e à Coordenação de Aperfeiçoamento de Pessoal de Nível Superior (CAPES), ao Conselho Nacional de Desenvolvimento Científico e Tecnológico (CNPq), à Financiadora de Estudos e Projetos (FINEP) e à Agência Nacional de Petróleo (ANP) pelo apoio financeiro. Os autores também agradecem à Petrobras pela autorização para a publicação deste trabalho.

\section{REFERÊNCIAS}

1. Mason, T. G.; Wilking, J. N.; Meleson, K.; Chang, C. B.; Graves, S. M.; J. Phys.: Condens. Matter 2006, 18, R635.

2. Solans, C.; Izquierdo, P.; Nolla, J.; Azemar, N.; Garcia-Celma, M. J.; Curr. Opin. Colloid Interface Sci. 2005, 10, 102.

3. Marcel, B. J.; Meinders, T. V.; Adv. Colloid Interface Sci. 2004, 108-109, 119.

4. Abismail, B.; Canselier, J. P.; Wilhelm, A. M.; Delmas, H.; Gourdon, C.; Ultrason. Sonochem. 1999, 6, 75.

5. Tadros, T.; Izquierdo, P.; Esquena, J.; Solans, C.; Adv. Colloid Interface Sci. 2004,108-109, 303.

6. Flourya, J.; Legrandb, J.; Desrumaux, A.; Chem. Eng. Sci. 2004, 59, 1285 .

7. Marie, P.; Perrier-Cornet, J. M.; Gervais, P.; J.-Food Eng. 2002, 53, 43.

8. Wu, W. H.; Tese de Doutorado, Pharmazie und Geowissenschaften der Albert-Ludwigs-Universität Freiburg, Alemanha, 2007.

9. Floury, J.; Desrumaux, A.; Axelos, M. A. V.; Legrand., J.; J. Food Eng. 2003, 58, 227.

10. Jafari, S. M.; He, Y.; Bhandari, B.; J. Food Eng. 2007, 82, 478.

11. Allured; Flavor and Fragrance Materials, Allured Publishing Corporation: Illinois, 1999, p. 545.

12. Zanin, S. M. W.; Miguel, M. D.; Imelli, M. C.; Oliveira, A. B.; Visão Acadêmica 2002, 3, 3.

13. Alexandridis, P.; Athanassiou, V.; Fukuda, S.; Hatton, T. A.; Langmuir 1994, 10, 2604

14. Moore, W. J.; Physical Chemistry, Prentice-Hall, Inc.: New Jersey, 1972.

15. Weirong, L.; Dejun, S.; Caifu, L.; Qian, L.; Jian, X.; J. Colloid Interface Sci. 2006, 303, 557. 